\title{
Phylogenetic Signal in Module Composition and Species Connectivity in Compartmentalized Host-Parasite Networks
}

\author{
Boris R. Krasnov, ${ }^{1, \star}$ Miguel A. Fortuna, ${ }^{2}$ David Mouillot, ${ }^{3,4}$ Irina S. Khokhlova, ${ }^{5}$ \\ Georgy I. Shenbrot, ${ }^{1}$ and Robert Poulin ${ }^{6}$
}

1. Mitrani Department of Desert Ecology, Swiss Institute for Dryland Environmental and Energy Research, Jacob Blaustein Institutes for Desert Research, Ben-Gurion University of the Negev, Sede Boqer Campus, 84990 Midreshet Ben-Gurion, Israel; 2. Department of Ecology and Evolutionary Biology, Princeton University, Princeton, New Jersey 08544; and Estación Biológica de Doñana (EBD-Consejo Superior de Investigaciones Científicas), Seville, Spain; 3. UMR 5119 CNRS-UM2-Institut Français de Recherche pour l'Exploitation de la Mer-Institut de Recherche pour le Développement Ecologie des Systèmes Marins Côtiers, Université Montpellier 2, Place Eugène Bataillon, 34095 Montpellier Cedex 05, France; 4. Australian Research Council Centre of Excellence for Coral Reef Studies, James Cook University, Townsville, Queensland 4811, Australia; 5. Wyler Department of Dryland Agriculture, French Associates Institute of Agriculture and Biotechnology of Drylands, Jacob Blaustein Institutes for Desert Research, Ben-Gurion University of the Negev, Sede Boqer Campus, 84990 Midreshet Ben-Gurion, Israel; 6. Department of Zoology, University of Otago, P.O. Box 56, Dunedin, New Zealand

Submitted August 9, 2011; Accepted November 29, 2011; Electronically published February 27, 2012

Online enhancement: appendix. Dryad data: http://dx.doi.org/10.5061/dryad.2q4p67m9.

ABSTRACT: Across different taxa, networks of mutualistic or antagonistic interactions show consistent architecture. Most networks are modular, with modules being distinct species subsets connected mainly with each other and having few connections to other modules. We investigate the phylogenetic relatedness of species within modules and whether a phylogenetic signal is detectable in the within- and among-module connectivity of species using 27 mammal-flea networks from the Palaearctic. In the 24 networks that were modular, closely related hosts co-occurred in the same module more often than expected by chance; in contrast, this was rarely the case for parasites. The within- and among-module connectivity of the same host or parasite species varied geographically. However, among-module but not within-module connectivity of host and parasites was somewhat phylogenetically constrained. These findings suggest that the establishment of host-parasite networks results from the interplay between phylogenetic influences acting mostly on hosts and local factors acting on parasites, to create an asymmetrically constrained pattern of geographic variation in modular structure. Modularity in host-parasite networks seems to result from the shared evolutionary history of hosts and by trait convergence among unrelated parasites. This suggests profound differences between hosts and parasites in the establishment and functioning of bipartite antagonistic networks.

Keywords: fleas, mammals, modules, networks, roles, phylogeny.

\footnotetext{
* Corresponding author; e-mail: krasnov@bgu.ac.il.
}

Am. Nat. 2012. Vol. 179, pp. 501-511. (c) 2012 by The University of Chicago. 0003-0147/2012/17904-53247\$15.00. All rights reserved. DOI: $10.1086 / 664612$

\section{Introduction}

In any ecosystem, species interact with each other in a variety of ways ranging from mutualism (e.g., plant-pollinator interactions) to antagonism (e.g., predator-prey or host-parasite interactions). Recently, these sets of interactions have been visualized as distinct natural entities called interaction networks, in which species are "nodes" connected to each other by "links" if they interact (see review in Dunne 2006; Montoya et al. 2006; Bascompte and Jordano 2007). There has been a huge increase in the number of studies of interaction networks during the last decade (e.g., Vázquez et al. 2005; Bascompte et al. 2006; Fortuna and Bascompte 2006; Burgos et al. 2007), although pioneering studies date back to the 1970s-1990s (May 1973; Hirata and Ulanovicz 1984, 1985; Paine 1992; Cohen 1994).

Despite sharp differences in the nature and topology of species interactions among ecological networks (e.g., mutualistic vs. antagonistic, and unipartite vs. bipartite), consistent patterns in network architecture have been revealed. For example, Kondoh et al. (2010) demonstrated that a nested structure characterizes both mutualistic networks and bipartite subwebs composing food webs. Furthermore, the number of interactions in any network varies among its component species with the majority of species having a few links only, while a minority of species interact with many other species (Jordano et al. 2003; Vázquez et al. 2005, 2007; Fortuna et al. 2010). In addition, many ecological networks appear to be modular (or compartmen- 
talized; Olesen et al. 2007; Dupont and Olesen 2009; Guimerà et al. 2010; Stouffer and Bascompte 2011). In a modularly structured network, species from some distinct subsets (called modules) are connected mainly with each other, while they have much fewer connections to other species belonging to other modules. Modular structure has been found to be ubiquitous in both ecological and nonecological networks (Girvan and Newman 2002; Amaral and Ottino 2004; Guimerà and Amaral 2005a).

The detection of modularity in numerous ecological networks (e.g., Olesen et al. 2007; Fortuna et al. 2010) has led to increasing interest in the species composition of modules. It has been shown that modules in food webs and mutualistic networks tend to be characterized by clusters of closely related species (Dupont and Olesen 2007; Mello et al. 2011) or species that converge on correlated sets of traits (Corbet 2000; Dicks et al. 2002). The latter is defined as a syndrome and is associated with the utilization of a specific group of mutualists (e.g., pollinators; see Fenster et al. 2004 for review). Moreover, module composition can also represent a combination of phylogenetic clusters and groups of species converging toward a syndrome (Olesen et al. 2007; Rezende et al. 2009; Donatti et al. 2011).

To the best of our knowledge, all studies of species composition of modules have been carried out for food webs and plant-animal mutualistic networks. In contrast, hostparasite networks have been neglected, although parasites represent a substantial portion of global biodiversity with $30 \%-50 \%$ of known animal species classified as parasites (Poulin and Morand 2004). Moreover, a high level of modularity with an important phylogenetic component in module composition have been predicted for host-parasite networks (mostly due to the high specificity of interactions) even before any empirical study of these networks using a modern approach (Levinsohn et al. 2006). Later studies indeed revealed a high level of modularity in hostparasite networks (Fortuna et al. 2010). However, the role of phylogeny in the module composition of host-parasite networks has never been studied. A strong phylogenetic component may be expected in host-parasite networks because phylogenetic closeness under the assumption of trait conservatism (Wiens et al. 2010), reflects phenotypic similarity (e.g., Brooks and McLennan 1991). As a result, certain lineages of parasites are more likely to exploit certain lineages of hosts so that a module might consist of closely related hosts and closely related parasites, although processes such as, for example, coevolutionary alternation may substantially change parasite preferences (Nuismer and Thompson 2006). In contrast, no phylogenetic signal could suggest random module composition likely based on opportunism or dispersal limitation, whereas a negative phylogenetic signal (i.e., closely related parasites mainly exploit distantly related hosts) might suggest complementarity within modules.

The topological properties of a species in a network such as its connectivity with species in its own module and with species from other modules have been shown to be affected by biological traits such as its degree of specialization or dispersal abilities (Olesen et al. 2007; Carstensen and Olesen 2009). In host-parasite networks, the number of links between a parasite species and its host species is determined by the parasite's host specificity (Poulin 2010). Host specificity, in turn, has been shown to be a true parasite species attribute in that it varies significantly more among parasite species than among populations within parasite species. Further, host specificity is to a large extent phylogenetically constrained (Krasnov et al. 2004), although in some taxa like fleas, this is true for some but not all biogeographic regions (Krasnov et al. 2011b). Similarly, the number of parasite species exploiting a host species in the networks used in this study tends to vary less within (across their geographic range) than among host species (Krasnov et al. 2005). Consequently, the within- and among-module connectivity of a host or parasite species in a network is expected to be $(a)$ a species-specific property, (b) phylogenetically constrained and, thus, $(c)$ geographically invariant. However, the variation in topological properties of the same species in different networks across its geographic range has never been studied. It is still unclear whether the within- and/or among-module connectivity of a given species differ among the different networks where it occurs.

Here, we examined the importance of phylogeny in determining the composition of modules and species connectivity in bipartite host-parasite networks of small mammals and their flea parasites from 27 distinct geographic regions of the Palaearctic. We test the hypothesis that phylogeny constrains the structure of bipartite networks across space by determining the composition of modules and the connectivity of species. To achieve this, we ask whether modules in each network consist of phylogenetically related host or parasite species or, alternatively, whether species within the same module represent random phylogenetic lineages. A strong phylogenetic signal in the species composition of modules would indicate shared evolutionary history instead of trait convergence in unrelated species as a process generating modularity. We also ask whether the within- and/or among-module connectivity of a host or parasite species is phylogenetically constrained and, hence, whether it is the same in a given species across geographically different networks. To answer these questions, we measured the phylogenetic signal in species connectivity within and across networks. Then, we evaluated the internetwork variation in connectivity of each widely distributed species by testing whether within- and among- 
module connectivity are repeatable within host or parasite species, that is, are less variable within than among species. In other words, we tested whether the values of connectivity are more similar among populations of the same species than among different species.

\section{Methods \\ Networks}

We used data from 27 published surveys carried out in different regions of the Palaearctic that reported parasite (fleas; Insecta: Siphonaptera) distribution on hosts (small mammals; Mammalia: Erinaceomorpha, Soricomorpha, Rodentia, and Lagomorpha; see appendix, available online). Fleas are hematophagous parasites that are most abundant and diverse on small mammals. Their life cycle is characterized by alternation between periods when they occur on the body of their hosts and periods when they occur in their hosts' burrows or nests. In most cases, larval fleas are not parasitic and develop entirely off-host in their burrow and/or nest. Flea species range from highly host specific to host opportunistic (Krasnov 2008). The data from each survey were organized as presence/absence matrices. It is commonly accepted that presence/absence rather than abundance matrices are more appropriate for the analyses of host-parasite networks because of $(a)$ the greater confidence they provide for scoring parasite occurrences as compared to parasite abundances (Gotelli and Rohde 2002) and (b) the aggregated character of parasite distributions (Poulin 2007).

\section{Modularity and Detection of Modules}

In order to identify nonoverlapping sets of hosts and parasites (i.e., modules) we used a simulated annealing (SA) optimization approach (Guimerà and Amaral $2005 a, 2005 b)$ for finding the maximization of a function called modularity ( $M$; Newman 2004; Newman and Girvan 2004) for each network. The equation for this function and its explanation are given elsewhere (e.g., Fortuna et al. 2010). The algorithm (implemented with the software NETCARTO, kindly provided by R. Guimerà) follows a heuristic procedure for maximizing the number of links within-modules and minimizing the number of links between modules. Due to its heuristic nature, we ran the algorithm 10 times for each network and used the maximum $M$ value obtained as the modularity of that network (Fortuna et al. 2010). The SA algorithm is considered as one of the most accurate algorithms for module detection (see Danon et al. 2005; Dupont and Olesen 2009 and references therein).

After estimating the value of modularity for a network, we tested whether this value departed significantly from random expectations. Null models intentionally exclude processes of interest and thus describe how the system would look like in the absence of these processes, allowing for randomization tests of ecological data (Gotelli 2000). The significance of the level of modularity was estimated using the fixed null model (Gotelli 2000; see Fortuna et al. 2010 for the rationale of selecting this null model). In this model, each random network has the same number of connections per species as the real network. Random networks ( $=$ matrices) were constructed using an independent swap algorithm in which the original matrix is reshuffled by repeatedly swapping $2 \times 2$ submatrices that preserve the row and column totals (Roberts and Stone 1990). The null model implemented in our study maintained the bipartite structure of the networks by allowing connections between hosts and parasites and not allowing connections among hosts or among parasites. The independent swap algorithm has been used to evaluate the significance of modularity in earlier studies (Guimerà and Amaral 2005a, 2005b; Olesen et al. 2007; Fortuna et al. 2010). Each random matrix was constructed by performing 10,000 random transpositions and using the MATLAB function swap.m written by B. Semmens (http://www.mathworks.com/matlabcentral/ fileexchange/3205-swap-m). We constructed 500 random matrices for each real network and calculated the degree of modularity $(M)$ for each of these matrices. We then calculated the statistical significance of the modularity of each observed network ( $p$ value) as the fraction of the 500 random matrices with a modularity value equal to or higher than the observed one. Further analyses were restricted only to those networks that were found to be significantly modular.

\section{Within- and Among-Module Connectivity}

In a modular ecological network, species interact mostly with other species within their own module but can also interact with a few species from other module(s). Topologically, therefore, a species, or node, can be characterized by (a) its standardized within-module degree $z$ (which describes how a species is connected to other species in the same module) and (b) its participation coefficient $P$ (which describes how a species is connected to species in other modules; Guimerà and Amaral 2005b). Note that the participation coefficient is referred to as among-module connectivity $c$ in Olesen et al. (2007). The withinmodule degree of a species is calculated as the difference between the within-module number of links of this species and the mean within-module number of links of all species in this module divided by the latter's standard deviation (Guimerà and Amaral 2005b). It indicates whether a spe- 
cies is more generalist (positive $z$ values) or more specialist (negative $z$ values) within the module than the other species in that module. The participation coefficient of a species is calculated as 1 minus the across-module sum of the squared quotients of that species' number of withinmodule links and that species' total number of links. It is therefore close to 1 if the links of a species are uniformly distributed among all the modules and 0 if all its links are within its own module. We calculated the participation coefficients for each species using NETCARTO (see above). Calculation of the within-module connectivity using NETCARTO does not take into account the bipartite nature of our networks. Consequently, we calculated the values of $z$ for each host and parasite species using the mean within-module number of links and its standard deviation for either hosts only or parasites only. Obviously, if a module contains only one host or parasite species or all species in a module have equal number of links, the within-module degree cannot be calculated due to the standard deviation of the within-module across-species number of links having a value of 0 . We assigned a $z$ value of 0 to these species because they are characterized by neither more nor less within-module connectivity than the average in their module. The results did not change when we reran the analyses after removal of these species. Calculation of the participation coefficients does not differ between unipartite and bipartite networks.

In the repeatability analyses (Arneberg et al. 1997), we analyzed the variation in $z$ and $P$ values for hosts and parasites separately by one-way ANOVAs in which species identity was the independent factor, using only species that occurred in at least two networks. A significant effect of species identity would indicate that within- and/or amongmodule connectivity is repeatable within species, that is, that $z$ and/or $P$ values for the same species from different geographic regions are more similar to each other than to values from other species. We estimated the proportion of the total variance originating from differences among species, as opposed to within species, following Sokal and Rohlf (1995).

\section{Phylogenetic Analyses}

To test whether host or flea species within a given module were represented by either closely or distantly related species or else by random assemblages of species belonging to different phylogenetic lineages, we determined whether there was a significant correlation between the phylogenetic distance of each species pair and their degree of cooccurrence within modules (Cavender-Bares et al. 2004). The phylogenetic distance was taken as the sum of the lengths of all branches linking two species in a phylogenetic tree, while pairwise values of co-occurrence were calcu- lated using Schoener's co-occurrence index (Schoener 1970). The significance of correlation coefficients was estimated by comparison of the observed coefficient with the mean of correlation coefficients computed for 999 randomized null matrices that maintained species occurrence frequency and number of species in a module using the independent swap algorithm. Reanalyses of these data using null models that randomly reshuffle phylogeny tip labels provided the same results.

We also tested for a phylogenetic signal in values of $z$ and $P$ of hosts and parasites within each network as well as after combining data for all host or parasite species across networks (i.e., creating phylogenetic trees for all host or all parasite species from all networks which showed modularity) using Blomberg et al.'s (2003) K. In the latter analysis, values of $z$ and $P$ for species that occurred in more than one network were averaged across networks. The rationale behind combining data across networks is that (a) the connectivity of a host or a parasite species in a network is determined by the number of its connections, that is number of parasite species infesting a host or number of host species used by a parasite, and $(b)$ each of these numbers has been shown to be a true attribute of a host or a parasite species and vary only within relatively narrow species-specific boundaries (Krasnov et al. 2004, 2005). Importantly, this was shown for roughly the same species of hosts and parasites that were used in this study.

Blomberg et al.'s (2003) K assumes a Brownian motion model of evolutionary process. Under this model, the evolutionary changes along branches are expected to have zero values. Their distribution is normal with a variance proportional to branch length (Felsenstein 1985). The $K$ term is calculated as the quotient of observed and expected mean square error (MSE) ratios. The observed ratio is the MSE of the tip data, measured from the phylogenetically correct means, divided by the MSE of the data calculated using the variance-covariance matrix derived from the tree. The expected ratio is computed from the phylogenetic tree and Brownian motion as a model for the evolutionary process. In other words, $K$ compares the observed phylogenetic signal in a trait to the signal under a Brownian motion model of trait evolution on a phylogeny (Blomberg et al. 2003). The higher the $K$ statistic, the stronger the phylogenetic signal in a trait. The $K$ term has a value of 1 for any trait in which Brownian motion is the true evolutionary model, which implies some degree of phylogenetic signal or conservatism. The $K$ values that do not differ significantly from 0 indicate no effect of phylogeny, whereas $K$ values greater than 1 indicate a strong phylogenetic signal and conservatism of traits. The statistical significance of the phylogenetic signal was evaluated by comparing observed patterns of the variance of independent contrasts of the trait to a null model involving shuf- 
fling species across the tips of the phylogenetic tree using 999 permutations (Kembel et al. 2010).

In addition, we analyzed the phylogenetic signal in $z$ and $P$ values together both within and across networks while substituting the original values of $z$ and $P$ with the scores calculated from principal component analyses of these two variables (composite variable F1). The resulting composite variables explained from $51.5 \%$ to $90.1 \%$ (on average, $72.7 \%$ ) of the variance for hosts and from $54.6 \%$ to $92.6 \%$ (on average, $72.5 \%$ ) of the variance for parasites; their eigenvalues were $1.03-1.81$ and 1.09-1.85, respectively. Correlations between the original values of $z$ and $P$ and values of the composite variable for hosts ranged from 0.71 and 0.95 , while those for parasites ranged from 0.74 and 0.96 . In all networks, correlations were positive except for correlations between values of $z$ and the composite variable in two networks for hosts (AK and EP) and two networks for parasites (KH and UV).

Phylogenetic analyses were carried out using the package "picante" (Kembel et al. 2010) implemented in R (R Development Core Team 2011). Phylogenetic trees for hosts and fleas were constructed based on trees of BinindaEmonds et al. (2007) for hosts and Whiting et al. (2008) for fleas with modifications explained in details in Krasnov et al. (2011a, 2011b, respectively).

\section{Results \\ Modularity and Modules}

Twenty-four of 27 networks were found to be significantly modular (table 1). In each of these networks, we detected from 3 to 10 modules that were composed of 1 to 9 host and 1 to 14 flea species.

The co-occurrence of species in the same modules correlated significantly with the phylogenetic distances among them in 16 of 24 networks for hosts and only 3 of 24 networks for fleas (table 1). Whenever the correlation between co-occurrence and phylogenetic distance was found significant, it was always negative and never positive, suggesting that closely related species co-occurred in the same module more often than expected by chance. An illustrative example with hosts from the eastern Balkhash desert (EB network) is presented in figure 1.

\section{Within- and Among-Module Connectivity of Hosts and Parasites}

Within networks, the within-module degree $(z)$ of host species ranged from -2.02 to 2.37 , while that of parasite species ranged from -2.80 to 2.27 . Values for the participation coefficient $(P)$ ranged from 0 to 0.78 in host species and from 0 to 0.80 in parasite species.
Table 1: Size ( $S$ : the sum of all host and flea species), the level of modularity ( $M$; see text for explanations), number of detected modules $(n M)$, correlation $(r)$ between co-occurrence of host and parasite species in the same module and the phylogenetic distance among them, and results of Blomberg et al.'s (2003) K test (see text for details) for phylogenetic signal in $P$ values in host and parasite species for flea-mammal networks

\begin{tabular}{|c|c|c|c|c|c|c|c|}
\hline \multirow[b]{2}{*}{ Network } & \multirow[b]{2}{*}{$S$} & \multirow[b]{2}{*}{$M$} & \multirow[b]{2}{*}{$n M$} & \multicolumn{2}{|c|}{ Correlation $(r)$} & \multicolumn{2}{|c|}{$\begin{array}{l}\text { Blomberg et } \\
\text { al.'s (2003) K }\end{array}$} \\
\hline & & & & Hosts & Parasites & Hosts & Parasites \\
\hline $\mathrm{AD}$ & 35 & $-.33 *$ & 4 & $-.30 *$ & .07 & .30 & .33 \\
\hline $\mathrm{AK}$ & 45 & $.32 *$ & 5 & .04 & .02 & .30 & $.49 *$ \\
\hline $\mathrm{AL}$ & 32 & .16 & $\ldots$ & $\ldots$ & $\ldots$ & $\ldots$ & $\ldots$ \\
\hline AR & 64 & $.37 *$ & 6 & -.10 & .06 & .26 & $.54 *$ \\
\hline $\mathrm{AZ}$ & 37 & $.42 *$ & 7 & $-.34 *$ & .05 & $.67 *$ & .31 \\
\hline DA & 37 & $.25^{*}$ & 5 & -.13 & .06 & .24 & .31 \\
\hline $\mathrm{EB}$ & 59 & $.51^{*}$ & 6 & $-.38 *$ & $-.10 *$ & .19 & $.41 *$ \\
\hline EP & 39 & $.30 *$ & 3 & $-.39 *$ & -.01 & .37 & .40 \\
\hline KH & 41 & $.33^{*}$ & 5 & $-.23 *$ & -.04 & .48 & .38 \\
\hline $\mathrm{KO}$ & 37 & $.22 *$ & 4 & $-.39 *$ & $-.12 *$ & .49 & $.49 *$ \\
\hline KR & 31 & .45 & $\ldots$ & $\ldots$ & $\ldots$ & $\ldots$ & $\ldots$ \\
\hline KU & 24 & $.23 *$ & 3 & .13 & .04 & .16 & .35 \\
\hline MU & 18 & .22 & $\ldots$ & $\ldots$ & $\ldots$ & $\ldots$ & $\ldots$ \\
\hline $\mathrm{MN}$ & 65 & $.39 *$ & 9 & -.10 & .01 & .32 & .28 \\
\hline MS & 35 & $.19 *$ & 5 & $-.21 *$ & -.01 & .18 & .60 \\
\hline MY & 49 & $.32 *$ & 8 & $-.24^{*}$ & -.06 & .37 & $.77 *$ \\
\hline NK & 50 & $.49 *$ & 6 & $-.17 *$ & .001 & $.43^{*}$ & .20 \\
\hline $\mathrm{NO}$ & 61 & $.30 *$ & 7 & $-.19 *$ & .06 & $.44^{*}$ & .31 \\
\hline $\mathrm{PO}$ & 49 & $.18^{*}$ & 4 & $-.21 *$ & -.09 & $.59 *$ & .19 \\
\hline SE & 22 & $.42 *$ & 3 & .12 & -.03 & $1.71 *$ & .52 \\
\hline SL & 41 & $.33 *$ & 5 & $-.2 *$ & $-.32 *$ & .21 & .28 \\
\hline TA & 58 & $.49 *$ & 10 & $-.2 *$ & -.02 & .32 & $.42 *$ \\
\hline TT & 53 & $.24 *$ & 6 & $-.23 *$ & .04 & $.37 *$ & .33 \\
\hline TU & 60 & $.24^{*}$ & 4 & $-.14^{*}$ & -.02 & .39 & .38 \\
\hline UV & 29 & $.24 *$ & 3 & .03 & -.03 & .48 & $.66^{*}$ \\
\hline VK & 65 & $.26^{*}$ & 7 & $-.17 *$ & .01 & $.39 *$ & .32 \\
\hline WS & 44 & $.23 *$ & 6 & -.10 & -.07 & .37 & .24 \\
\hline
\end{tabular}

Note: Significance of modularity was tested against that of fixed null models. Significance of correlation between co-occurrence of host and parasite species in the same module and the phylogenetic distance among them was tested with respect to randomizations. Significance of Blomberg et al.'s (2003) $K$ was determined using the quantiles of the observed phylogenetically independent contrast variance versus the null distribution, which are used to test for greater phylogenetic signal than expected (Kembel et al. 2010). Asterisk indicates $p<.05$ ). See appendix, available online, for abbreviations of network names.

No significant phylogenetic signal in $z$ values was detected for either hosts or parasites within any network $(K=0.07-0.68$ and $K=0.18-1.03$, respectively; $p>.10$ for all). The same was true for the composite variable F1 ( $K=0.11-0.77$ for hosts and $K=0.25-0.87$ for parasites; $p>.20$ for all). In contrast, a significant phylogenetic signal in $P$ values was found in seven networks for hosts and seven other networks for parasites (table 1). Significance 


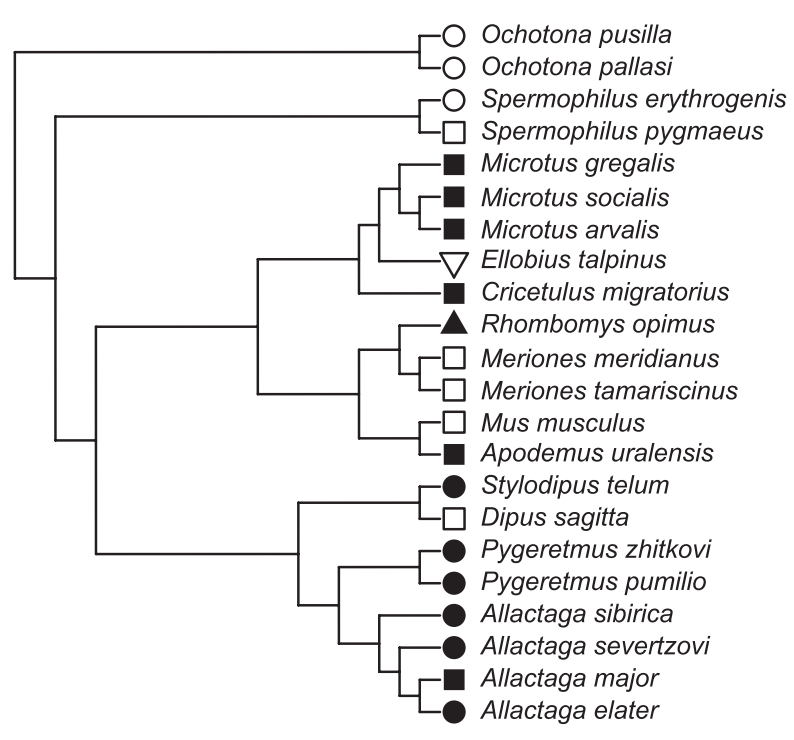

Figure 1: Phylogenetic tree of host species from the flea-mammal network of the eastern Balkhash desert with their occurrences in a particular module mapped on the tips of the tree. Identical symbols denote occurrences of species in the same module.

of these results was supported by meta-analysis using Fisher's combined probability test $\left(\chi^{2}=84.6\right.$ for hosts and $\chi^{2}=83.0$ for parasites; $p<.001$ for both). Across all host or parasite species and across networks, we detected significant phylogenetic signals in $P$ values $(K=0.16$ and $K=0.21 ; p<.05$ for both), but not in either $z$ values ( $K=0.04$ for hosts and $K=0.18$ for parasites; $p>.20$ for both) or the composite variable F1 $(K=0.07$ for hosts and $K=0.19$ for parasites; $p>.25$ for both). In other words, across networks, and at least in some networks, closely related hosts and parasites demonstrated more similarity in among-module connectivity than expected by chance (i.e., in the case of basal polytomy). An illustrative example with parasites from the Moyyunkum desert (MO network) is presented in figure 2.

Seventy-two of 121 host and 125 of 209 flea species from 24 modular networks occurred in more than one network (2-20 and 2-18 networks per species, respectively). The repeatability analysis for these species demonstrated that within- and among-module connectivity were repeatable within the same species (table 2). The significant species effect suggests that $z$ and $P$ values of the same species from different regions are more similar to each other than expected by chance and vary significantly among species. However, the percentage of the variation among samples accounted for by differences among species, as opposed to within taxa, was not especially high (table 2). This suggests the existence of substantial geographic variation in connectivity within species.

\section{Discussion}

The vast majority of host-parasite networks considered here are significantly modular. This is not especially surprising because many antagonistic (including host-parasite) networks have previously been reported as modular (e.g., Prado and Lewinsohn 2004; Fortuna et al. 2010). Moreover, higher modularity in antagonistic as compared to mutualistic networks has recently been proved (Thebault and Fontaine 2010; Fontaine et al. 2011). However, beyond reemphasizing the modularity of host-parasite networks, this study uncovered three novel patterns. First, in many host-parasite networks, closely related hosts tended to cluster in the same modules, while the distribution of parasite lineages among modules did not differ from random. Second, the within- and among-module connectivity of either host or parasite species may differ in different networks, that is, it may vary geographically. Third, the among-module but not within-module connectivity of host and parasite species appeared to be to some extent phylogenetically constrained.

\section{Modules Contain Phylogenetically Clustered Hosts but Not Parasites}

Phylogenetic clustering in module composition has been detected in ecological networks. For example, nonrandom associations between plants and insects in plant-pollinator networks suggest that these assemblages exert reciprocal selection on each other (Dupont and Olesen 2007; Olesen et al. 2007). Also, module composition in frugivorous batsfruit plants networks follows genus-genus associations (Mello et al. 2011). Finally, phylogenetic signals associated with compartments have been reported in food webs (Rezende et al. 2009) and mutualistic networks (Donatti et al. 2011).

In a modular host-parasite network, hosts from the same module are exploited by roughly the same parasite assemblage. One reason for this pattern may be the similarity of these hosts as resources for parasites. In other words, hosts from the same module should be similar in (a) the nature (e.g., chemical composition) or (b) the availability of the resources for parasites, and/or $(c)$ their antiparasitic defenses. In the host-parasite networks studied here, hosts provide two main resources for parasites: blood as nutrition for adult fleas and a burrow/nest environment for the development of eggs, larvae, and pupae. Obviously, parasites exploit only hosts with $(a)$ blood that satisfies their requirements and that can be easily extracted, $(b)$ behavioral and immunological defenses that can be overcome, and $(c)$ a burrow suitable for the development of preimago. Host species differ in these preconditions (see review in Krasnov 2008), while parasites differ in their 


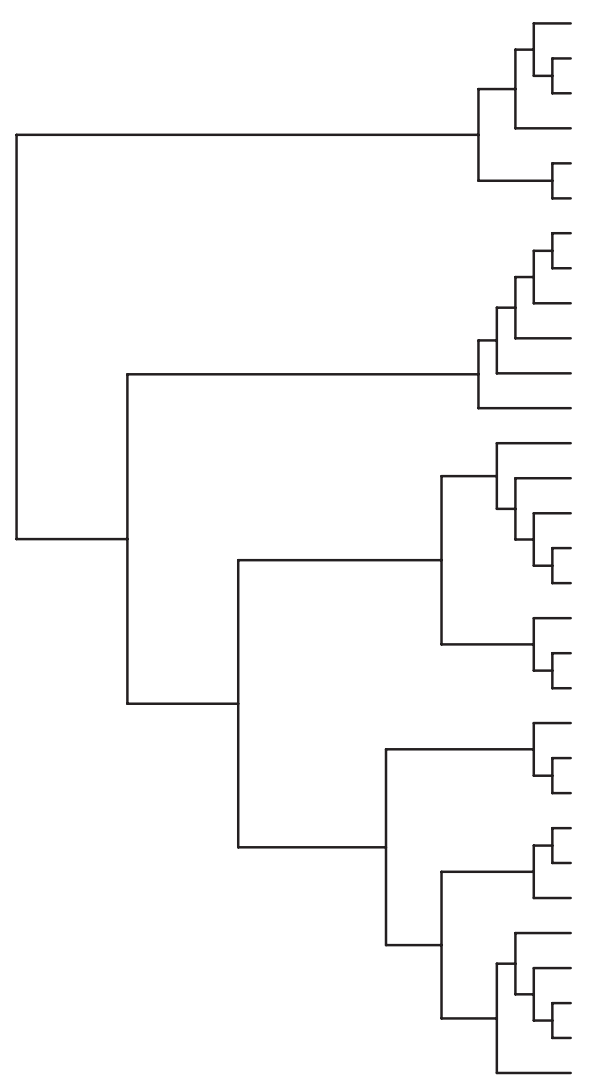

Rhadinopsylla cedestis

Stenoponia vlasovi

Stenoponia conspecta

Neopsylla setosa

Coptopsylla lamellifer

Ctenophthalmus dolichus

Xenopsylla skrjabini

Xenopsylla conformis

Xenopsylla gerbilli

Xenopsylla hirtipes

Synosternus longispinus

Echidnophaga oschanini

Desertopsylla rothschildi

Mesopsylla lenis

Mesopsylla eucta

Mesopsylla tuschkan

Mesopsylla hebes

Paradoxopsyllus repandus .

Ophthalmopsylla volgensis .

Frontopsylla macrophthalma

Amphipsylla rossica

Leptopsylla nana

Leptopsylla segnis

Citellophilus trispinus

Oropsylla ilovaiskii

Rostropsylla daca

Nosopsyllus tersus

Nosopsyllus laeviceps

Nosopsyllus turkmenicus

Nosopsyllus aralis

Nosopsyllus fidus

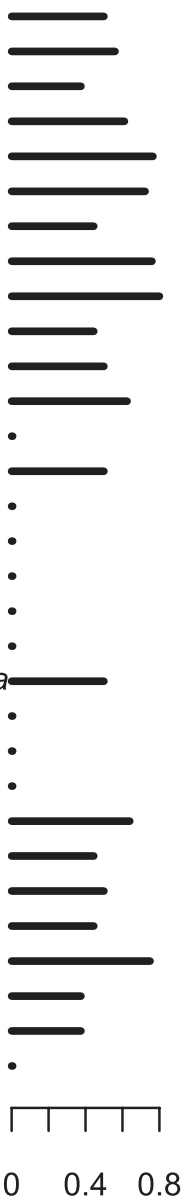

Participation coefficient

Figure 2: Participation coefficient, or $P$, values of parasite species from the flea-mammal network of the Moyyunkum desert plotted against their phylogenetic positions (created using package "ape" implemented in R software environment; Paradis 2006).

abilities to exploit hosts that differ in these key properties. Consequently, parasites with similar requirements are expected to exploit hosts that are similar with respect to those traits. In general, phylogenetic relatedness predicts overall life-history and ecological similarity among species (Brooks and McLennan 1991; Silvertown et al. 1997). Therefore, closely related host species should be more similar in all relevant characters than phylogenetically distant hosts (e.g., Galbe and Oliver 1992; Mooring et al. 2004). As a result, closely related hosts may have roughly the same set of parasites and, thus, comprise a substantial portion of the species in a module. However, correlations between host phylogenetic distance and their co-occurrence in modules were relatively weak, suggesting that modules do not comprise only closely related hosts. This can be reconciled with $(a)$ the limiting similarity of some of closely related hosts (Tilman 1982; Abrams 1983; Sil- vertown 2004) and (b) the fact that parasites can exploit distantly related hosts due to host switching, ecological fitting or coevolutionary alternation (see below).

In sharp contrast, the distribution of parasite phylogenetic lineages among modules did not differ from random expectations. Several non-mutually exclusive processes may explain this host-parasite asymmetry. First, the history of many parasites is characterized by frequent switching between related and unrelated host lineages (Krasnov and Shenbrot 2002; Zietara and Lumme 2002; Johnson et al. 2011). Second, this can be the outcome of ecological fitting, which occurs when $(a)$ distantly related parasites specialize on a resource that is widespread among many host species (i.e., blood) and $(b)$ a parasite mainly tracks this resource rather than a host lineage per se (Janzen 1985; Brooks et al. 2006). Hence, the similarity in parasite assemblages between host species can be deter- 
Table 2: Summary of the repeatability analyses (ANOVAs) of $z$ and $P$ values across host and parasite species from 24 networks $(p<.01$ for all).

\begin{tabular}{lccrc}
\hline & Measure of & & & $\begin{array}{c}\text { \% variation accounted } \\
\text { by difference among } \\
\text { species }\end{array}$ \\
\hline Hosts/parasites & $z$ & $F$ & df & 17.6 \\
Connectivity & 2.1 & 74,305 & 19.5 \\
& $P$ & 2.2 & 74,305 & 7.6 \\
& $P$ & 1.4 & 122,445 & 16.3 \\
\hline
\end{tabular}

mined by their physiological or ecological similarity independently of their phylogeny. Third, coevolutionary alternation (Thompson 2005; Nuismer and Thompson 2006) may result in cycles of evolutionary change in parasite preferences for host species. This may happen if, at any given time, selection favors parasites that exploit the host with the lowest defensive abilities. Frequent attacks would select for increased defense in this host, whereas decreased defense would be selected in rarely attacked host species because resistance is costly (Tella et all. 2002; Poulin 2007). In addition to these three processes, similar traits related to resource acquisition evolve independently in parasites from different lineages that exploit the same hosts (Poulin 2007; Pérez-Losada et al. 2009). For example, one of the most prominent adaptations to parasitism in fleas consists of sclerotinized structures such as helmets, ctenidia, spines, and setae that anchor the flea within host hair to resist grooming. These features correlate with particular characteristics of the host's hair and have been suggested to evolve convergently in different flea lineages (Traub 1985; Medvedev 2005).

\section{Host and Parasite Connectivity in a Network: Phylogenetic and Geographic Patterns}

Within-module connectivity values indicated that some host and parasite species were more generalist within their modules than the average (positive $z$ values), while others were more specialist than the average (negative $z$ values). Among-module connectivity values demonstrated that some hosts were infected by parasites from other modules ( $P$ values close to 1$)$, while others were infected only by parasites from their module ( $P$ values equal or close to $0)$. Similarly, some parasites exploited hosts from both their own and other modules, whereas other parasites attacked host from their own module only.

Within a host-parasite network, species were characterized by any combination of within- and among-module connectivity. For example, some species have few links inside their own module and even fewer to other modules. Among hosts, these species harbor species-poor parasite assemblages represented mainly by host-specific parasites.
Among parasites, these species are highly host specific. Given a tendency for host phylogenetic clustering in the modules, the host spectrum of such parasites is limited to few host phylogenetic lineages. The parasite assemblages harbored by a host, when species poor, comprised parasites recorded on a variety of other hosts. Furthermore, a parasite might be host specific in terms of the size of its host spectrum but not in terms of its hosts' phylogenetic diversity. Finally, a species can be a generalist in relation to the number of both within- and among-module links. Such hosts harbor large and diverse parasite assemblages, while such parasites exploit unrelated host species.

The connectivity of a host in a bipartite network corresponds to the number of parasites that exploit it. This number is a true host species attribute, possibly because species-specific thresholds of host defense against parasites limit their ability to cope with multiple parasites (Krasnov et al. 2005). The phylogenetic signal found for amongmodule connectivity of hosts in some networks and across all species supports this. However, (a) no phylogenetic signal in within-module connectivity was found, $(b)$ a phylogenetic signal in among-module connectivity was found in less than a half the networks, $(c)$ it was found mainly on continental scales; and $(d)$ the percentage of variation in among-module connectivity among host samples associated with differences between host species was rather low. This suggests that, despite some phylogenetic dependence in among-module connectivity, the latter can also be strongly affected by local conditions. Indeed, investigations of various host-parasite associations show that the diversity of parasite assemblages in the same host is affected by local factors such as the availability of phylogenetically related host species and climatic conditions (Fellis and Esch 2005; Goater et al. 2005; Krasnov et al. 2005).

Similarly, the among-module connectivity of parasites in a network is determined by both the size and phylogenetic diversity of their host spectrum. Both these measures of host specificity are true attributes of a parasite species, although they vary spatially due to local factors (Krasnov et al. 2004, 2008; Korallo-Vinarskaya et al. 2009). A relationship between specificity and local ecological fac- 
tors has been reported for different victim-exploiter associations (Smiley 1978; Funk and Bernays 2001) and is sometimes seen as the main determinant of host specialization (Smiley 1978). Geographic variation in the level of specialization has also been found for mutualistic associations (Olesen and Jordano 2002). In addition, a phylogenetic signal for host specificity among fleas has been found at a continental but not local scale (Krasnov et al. 2011b). Geographic variation in host specificity and the scale dependence of the phylogenetic signal in this trait might explain why we only detected phylogenetic influences on among-module connectivity in parasites in very few networks.

In addition, the reason behind the lack of a phylogenetic signal in within-module connectivity is, at least for hosts, a strong phylogenetic signal in the modular structure and the scattered distribution of species with high $z$ values among modules. Therefore, if there are only few species with high $z$ values in each module, they should be phylogenetically separated. Overall, the implications of phylogenetic signals for network structure are important. For instance, with a strong phylogenetic signal in among-module connectivity, the extinction of certain evolutionary lineages with high among-module connectivity would fragment a network into isolated modules. If, however, the phylogenetic signal in within-module connectivity is strong, then the same perturbation would lead to fragmentation of modules (Donatti et al. 2011).

\section{Conclusions}

Our results suggest that modular composition and species connectivity in host-parasite networks represent an asymmetric interplay between phylogenetic constraints and local conditions. The among-module connectivity of host and parasite species in a network is both phylogenetically constrained and affected to a certain extent by local factors. The modular structure of host-parasite networks is influenced by host phylogeny but not by parasite phylogeny $\rightarrow$ This is despite the much closer phylogenetic relatedness among parasites (all in order Siphonaptera) than among hosts (several unrelated mammalian orders) in our networks, thus stressing the robustness of the host-parasite asymmetry. The significant co-occurrence of phylogenetically related hosts but not parasites in the same module: $\rightarrow$ suggests that modularity in host-parasite networks is generated by the shared evolutionary history of hosts and by trait convergence in unrelated parasite species. Similarly, Fonseca and Ganade (1996) found that phylogenetically related myrmecophytes were exploited by the same an+ species belonging to different taxa, suggesting that host identity is an important force structuring plant-ant networks. Furthermore, the phylogenetic randomness in th $\rightarrow$ parasite composition of modules implies that it is driven mainly by abundance or dispersal within a region, whereas the phylogenetic signal in the host composition of modules suggests that modules follow deterministic associations underpinned by phylogeny. These patterns suggest profound differences between hosts and parasites in the establishment and functioning of bipartite antagonistic networks. The more predictable host component of network architecture may be particularly useful to forecast the consequences of global change and species range shifts on the spread of vector-borne diseases.

\section{Acknowledgments}

We thank R. Guimerà for providing the NETCARTO software and J. Meiler and L. Prigozhin for help with MATLAB commands. We thank two anonymous reviewers for their most helpful comments on an earlier version of the manuscript. This study was partly supported by the Israel Science Foundation (grant 27/08 to for B.R.K. and I.S.K.) and by a Marie Curie International Outgoing Fellowship within the Seventh European Community Framework Programme (to M.A.F.). This is publication 738 of the Mitrani Department of Desert Ecology.

\section{Literature Cited}

Abrams, P. 1983. The theory of limiting similarity. Annual Review of Ecology and Systematics 14:359-376.

Amaral, L. A. N., and J. M. Ottino. 2004. Complex networks: completing the framework for the study of complex systems. European Physics Journal 38:47-162.

Arneberg, P., A. Skorping, A., and A. F. Read. 1997. Is population density a species character? comparative analyses of the nematode parasites of mammals. Oikos 80:289-300.

Bascompte, J., and P. Jordano. 2007. Plant-animal mutualistic networks: the architecture of biodiversity. Annual Review of Ecology, Evolution, and Systematics 38:567-593.

Bascompte, J., P. Jordano, and J. M. Olesen. 2006. Asymmetric coevolutionary networks facilitate biodiversity maintenance. Science 312:431-433.

Bininda-Emonds, O. R. P., M. Cardillo, K. E. Jones, R. D. E. MacPhee, R. M. D. Beck, R. Grenyer, S. A. Price, R. A. Vos, J. L. Gittleman, and A. Purvis. 2007. The delayed rise of present-day mammals. Nature 446:507-512.

Blomberg, S. P., T. Garland Jr., and A. R. Ives. 2003. Testing for phylogenetic signal in comparative data: behavioral traits are more labile. Evolution 57:717-745.

Brooks, D. R. and McLennan, D. A. (1991). Phylogeny, ecology, and behaviour: a research program in comparative biology. University of Chicago Press, Chicago.

Brooks, D. R., V. León-Règagnon, D. A. McLennan, and D. Zelmer. 2006. Ecological fitting as a determinant of the community structure of platyhelminth parasites of anurans. Ecology 87:S76-S85.

Burgos, E., H. Ceva, R. P. J. Perazzo, M. Devoto, D. Medan, M. 


\section{The American Naturalist}

Zimmermann, and A. Maria Delbue. 2007. Why nestedness in mutualistic networks? Journal of Theoretical Biology 249:307-313.

$\rightarrow$ Carstensen, D. W., and J. M. Olesen. 2009. Wallacea and its nectar $\rightarrow$ ivorous birds: nestedness and modules. Journal of Biogeography 36:1540-1550.

$\rightarrow$ Cavender-Bares, J., D. D. Ackerly, D. A. Baum, and F. A. Bazzaz. 2004. Phylogenetic overdispersion in Floridian oak communities $\rightarrow$ American Naturalist 163:823-843.

Cohen, J. E. 1994. Marine and continental food webs: three para $\rightarrow$ doxes? Philosophical Transactions of the Royal Society B: biological Sciences 343:57-69.

$\rightarrow$ Corbet, S. A. 2000. Conserving compartments in pollination webs. Conservation Biology 14:1229-1231.

$\rightarrow$ Dicks, L. V., S. A. Corbet, and R. F. Pywell. 2002. Compartmental $\rightarrow$ ization in plant-insect flower visitor webs. Journal of Animal Ecology 71:32-43.

$\rightarrow$ Danon, L., J. Duch, A. Díaz-Guilera, and A. Arenas. 2005. Comparing community structure identification. Journal of Statistical Mechanics: Theory and Experiments 1:P09008.

$\rightarrow$ Donatti, C. I., P. R. Guimarães, M. Galetti, M. A. Pizo, F. M. D. Marquitti, and R. Dirzo. 2011. Analysis of a hyper-diverse seed dispersal network: modularity and underlying mechanisms. Ecol $\rightarrow$ ogy Letters 14:773-781.

Dunne, J. A. 2006. The network structure of food webs. Pages 2786 in M. Pacual and J. A. Dunne, eds. Ecological networks: linkin $\rightarrow$ structure to dynamics in food webs. Oxford University Press $\rightarrow$ J Oxford.

$\rightarrow$ Dupont, Y. L., and J. M. Olesen. 2009. Ecological modules and roles of species in heathland plant-insect flower visitor networks. Journal of Animal Ecology 78:346-353.

$\rightarrow$ Fellis, K. J., and G. W. Esch. 2005. Autogenic-allogenic status affects interpond community similarity and species area relationship of macroparasites in the bluegill sunfish, Lepomis macrochirus, fron $\rightarrow$ a series of freshwater ponds in the Piedmont area of North Carolina. Journal of Parasitology 91:764-767.

$\rightarrow$ Felsenstein, J. 1985. Phylogenies and the comparative method. American Naturalist 125:1-15.

$\rightarrow$ Fenster, C. B., W. S. Armbruster, P. Wilson, M. Dudash, and J. D. Thomson. 2004. Pollination syndromes and floral specialization $\rightarrow$ Annual Review of Ecology, Evolution and Systematics 35:375-403.

$\rightarrow$ Fonseca, C. R., and Ganade, G. 1996. Asymmetries, compartments and null interactions in an Amazonian ant-plant community. Journal of Animal Ecology 65:339-347.

$\rightarrow$ Fontaine, C., P. R. Guimarães, S. Kéfi, N. Loeuille, J. Memmott, W. H. van der Putten, F. J. F. van Veen, and E. Thébault. 2011. The ecological and evolutionary implications of merging different type of networks. Ecology Letters 14:1170-1181.

$\rightarrow$ Fortuna, M. A., and J. Bascompte. 2006. Habitat loss and the structure of plant-animal mutualistic networks. Ecology Letters 9:281-286

$\rightarrow$ Fortuna, M. A., D. B. Stouffer, J. M. Olesen, P. Jordano, D. Mouillot, B. R. Krasnov, R. Poulin, and J. Bascompte. 2010. Nestedness versus modularity in ecological networks: two sides of the same coin? Journal of Animal Ecology 79:811-817.

$\rightarrow$ Funk, D. J., and E. A. Bernays. 2001. Geographic variation in host specificity reveals host range evolution in Uroleucon ambrosiae aphids. Ecology 82:726-739.

Galbe, J., and J. H. Oliver. 1992. Immune response of lizards anc $\rightarrow$ rodents to larval Ixodes scapularis (Acari, Ixodidae). Journal of Medical Entomology 29:774-783.

$\rightarrow$ Girvan, M., and M. E. J. Newman. 2002. Community structure in social and biological networks. Proceedings of the National Academy of Sciences of the USA 99:7821-7826.

Goater, C. P., R. E. Baldwin, and G. J. Scrimgeour. 2005. Physicochemical determinants of helminth component community structure in whitefish (Coregonus clupeaformes) from adjacent lakes in northern Alberta, Canada. Parasitology 131:713-722.

Gotelli, N. J. 2000. Null model analysis of species co-occurrence patterns. Ecology 81:2606-2621.

Gotelli, N. J., and K. Rohde. 2002. Co-occurrence of ectoparasites of marine fishes: a null model analysis. Ecology Letters 5:86-94.

$\rightarrow$ Guimerà, R., and L. A. N. Amaral. 2005a. Cartography of complex networks: modules and universal roles. Journal of Statistical Mechanics: Theory and Experiment 1:P02001.

$-2005 b$. Functional cartography of complex metabolic networks. Nature 433:895-900.

$\rightarrow$ Guimerà, R., D. B. Stouffer, M. Sales-Pardo, E. A. Leicht, M. E. J. Newman, and L. A. N. Amaral. 2010. Origin of compartmentalization in food webs. Ecology 91:2941-2951.

$\rightarrow$ Hirata, H., and R. E. Ulanovicz. 1984. Information theoretical analysis of ecological networks. International Journal of Systems Science 15:261-270.

$\rightarrow$ 1985. Information theoretical analysis of the aggregation and hierarchical structure of ecological networks. Journal of Theoretical Biology 116:321-341.

Janzen, D. H. 1985. On ecological fitting. Oikos 45:308-310.

Johnson, K. P., J. D. Weckstein, J. M. Meyer, and D. H. Clayton. 2011. There and back again: switching between host orders by avian body lice (Ischnocera: Goniodidae). Biological Journal of the Linnean Society 102:614-625.

$\rightarrow$ Jordano, P., J. Bascompte, and J. M. Olesen. 2003. Invariant properties in coevolutionary networks of plant-animal interactions. Ecology Letters 6:69-81.

Kembel, S. W., P. D. Cowan, M. R. Helmus, W. K. Cornwell, H. Morlon, D. D. Ackerly, S. P. Blomberg, and C. O. Webb. 2010. Picante: $\mathrm{R}$ tools for integrating phylogenies and ecology. Bioinformatics 26:1463-1464.

$\rightarrow$ Kondoh, M., S. Kato, and Y. Sakato. 2010. Food webs are built up with nested subwebs. Ecology 91:3123-3130.

Korallo-Vinarskaya, N. P., B. R. Krasnov, M. V. Vinarski, G. I. Shenbrot, D. Mouillot, and R. Poulin. 2009. Stability in abundance and niche breadth of gamasid mites across environmental conditions, parasite identity and host pools. Evolutionary Ecology 23:329-345.

Krasnov, B. R. 2008. Functional and evolutionary ecology of fleas: a model for ecological parasitology. Cambridge University Press, Cambridge.

Krasnov, B. R., and G. I. Shenbrot. 2002. Coevolutionary events in history of association of jerboas (Rodentia: Dipodidae) and their flea parasites. Israel Journal of Zoology 48:331-350.

Krasnov, B. R., D. Mouillot, G. I. Shenbrot, I. S. Khokhlova, and R. Poulin. 2004. Geographical variation in host specificity of fleas (Siphonaptera): the influence of phylogeny and local environmental conditions. Ecography 27:787-797

$\rightarrow$ Krasnov, B. R., G. I. Shenbrot, D. Mouillot, I. S. Khokhlova, and R. Poulin. 2005. Spatial variation in species diversity and composition of flea assemblages in small mammalian hosts: geographic distance or faunal similarity? Journal of Biogeography 32:633-644.

Krasnov, B. R., G. I. Shenbrot, I. S. Khokhlova, D. Mouillot, and R. Poulin. 2008. Latitudinal gradients in niche breadth: empirical evidence from haematophagous ectoparasites. Journal of Biogeography 35:592-601. 
Krasnov, B. R., M. Stanko, I. S. Khokhlova, G. I. Shenbrot, S. Morand, N. P. Korallo-Vinarskaya, and M. V. Vinarski. 2011a. Nestedness and beta diversity in ectoparasite assemblages of small mammalian hosts: effects of parasite affinity, host biology and scale. Oikos 120 630-639.

$\rightarrow$ Krasnov, B. R., R. Poulin, and D. Mouillot. 2011b. Scale dependence of phylogenetic signal in ecological traits of ectoparasites. Ecog raphy 34:114-122.

$\rightarrow$ Lewinsohn, T. M., P. I. Prado, P. Jordano, J. Bascompte, and J. M $\rightarrow$ Olesen. 2006. Structure in plant-animal interaction assemblages. Oikos 113:174-184.

May, R. M. 1973. Stability and complexity in model ecosystems. Princeton University Press, Princeton, NJ.

Medvedev, S. G. 2005. An attempted system analysis of the evolution of the order of fleas (Siphonaptera). Lectures in memoriam N. A. Kholodkovsky, no. 57. Russian Entomological Society and Zoo logical Institute of Russian Academy of Sciences, Saint Petersburg. [In Russian.]

$\rightarrow$ Mello, M. A. R., F. M. D. Marquitti, P. R. Guimarães, E. K. V. Kalko, P. Jordano, and M. A. M. de Aguiar. 2011. The missing part $\mathrm{O}_{\mathbf{1}}$ seed dispersal networks: structure and robustness of bat-fruit interactions. PLoS ONE 6:e17395.

$\rightarrow$ Montoya, J. M., S. L. Pimm, and R. V. Sole. 2006. Ecological networks $\rightarrow$ and their fragility. Nature 442:259-269.

$\rightarrow$ Mooring, M. S., D. T. Blumstein, and C. J. Stoner. 2004. The evolution of parasite-defense grooming in ungulates. Biological Journal of the Linnean Society 81:17-37.

$\rightarrow$ Nuismer, S. L., and J. N. Thompson. 2006. Coevolutionary alternation in antagonistic interactions. Evolution 60:2207-2217.

Newman, M. E. J. 2004. Fast algorithm for detecting community structure in networks. Physical Review E69:P066133.

Newman, M. E. J., and M. Girvan. 2004. Finding and evaluating community structure in networks. Physical Review E69:P026113.

$\rightarrow$ Olesen, J., and P. Jordano. 2002. Geographic patterns in plant-pollinator mutualistic networks. Ecology 83:2416-2424.

$\rightarrow$ Olesen, J. M., J. Bascompte, Y. L. Dupont, and P. Jordano. 2007. The modularity of pollination networks. Proceedings of the National Academy of Sciences of the USA 104:19891-19896.

$\rightarrow$ Paine, R. T. 1992. Food-web analysis through field measurement of per capita interaction strength. Nature 355:73-75.

Paradis, E. 2006. Analyses of phylogenetics and evolution with R. Springer, New York.

Pérez-Losada, M., J. T. Høeg, and K. A. Crandall. 2009. Remarkablr convergent evolution in specialized parasitic Thecostraca (Crustacea). BMC Biology 7:15, doi:10.1186/1741-7007-7-15.

Poulin, R. 2007. Evolutionary ecology of parasites: from individual to communities. 2nd ed. Princeton University Press, Princeton, NJ.

$\rightarrow-$ 2010. Network analysis shining light on parasite ecology and diversity. Trends in Parasitology 26:492-498.

Poulin, R., and S. Morand. 2004. Parasite biodiversity. Smithsonian Institution, Washington, DC.

$\rightarrow$ Prado, P. I. and T. M. Lewinsohn. 2004. Compartments in insectplant associations and their consequences for community structure. Journal of Animal Ecology 73:1168-1178.
R Development Core Team. 2011. R: a language and environment for statistical computing. R Foundation for Statistical Computing, Vienna, Austria. http://www.R-project.org/.

Rezende, E. L., E. M. Albert, M. A. Fortuna, and J. Bascompte. 2009. Compartments in a marine food web associated with phylogeny, body mass, and habitat structure. Ecology Letters 12:779-788.

Roberts, A., and L. Stone. 1990. Island-sharing by archipelago species. Oecologia (Berlin) 83:560-567.

Schoener, T. W. 1970. Nonsynchronous spatial overlap of lizards in patchy habitats. Ecology 51:408-418.

$\rightarrow$ Silvertown, J. 2004. Plant coexistence and the niche. Trends in Ecology \& Evolution 19:605-611.

Silvertown, J., M. Franco, and J. L. Harper, eds. 1997. Plant life histories: ecology, phylogeny and evolution. Cambridge University Press, New York.

Smiley, J. 1978. Plant chemistry and the evolution of host specificity: new evidence from Heliconius and Passiflora. Science 201:745-747.

Sokal, R. R., and F. J. Rohlf. 1995. Biometry. 3rd ed. Freeman, New York.

Stouffer, D. B., and J. Bascompte. 2011. Compartmentalization increases food-web persistence. Proceedings of the National Academy of Sciences of the USA 108:3648-3652.

Tella, J. L., A. Scheuerlein, and R. E. Ricklefs. 2002. Is cell-mediated immunity related to the evolution of life-history strategies in birds? Proceedings of the Royal Society B: Biological Sciences 269:10591066.

Thebault, E., and C. Fontaine. 2010. Stability of ecological communities and the architecture of mutualistic and trophic networks. Science 329:853-856.

Thompson, J. N. 2005. The geographic mosaic of coevolution. University of Chicago Press, Chicago.

Tilman, D. 1982. Resource competition and community structure. Princeton University Press, Princeton, NJ.

Traub, R. 1985. Coevolution of fleas and mammals. Pages 295-437 in K. C. Kim, ed. Coevolution of parasitic arthropods and mammals. Wiley, New York.

Vázquez, D. P., R. Poulin, B. R. Krasnov, and G. I. Shenbrot. 2005. Species abundance and the distribution of specialization in hostparasite interaction networks. Journal of Animal Ecology 74:946955.

$\rightarrow$ Vázquez, D. P., C. J. Melián, N. M. Williams, N. Blüthgen, B. R. Krasnov, and R. Poulin. 2007. Species abundance and asymmetric interaction strength in ecological networks. Oikos 116:1120-1127.

Whiting, M. F., A. S. Whiting, M. W. Hastriter, and K. Dittmar. 2008. A molecular phylogeny of fleas (Insecta: Siphonaptera): origins and host associations. Cladistics 24:677-707.

Wiens, J. J., D. D. Ackerly, A. P. Allen, B. L. Anacker, L. B. Buckley, H. V. Cornell, E. I. Damschen, et al. 2010. Niche conservatism as an emerging principle in ecology and conservation biology. Ecology Letters 13:1310-1324.

Zietara, M. S., and J. Lumme. 2002. Speciation by host switch and adaptive radiation in a fish parasite genus Gyrodactylus (Monogenea, Gyrodactylidae). Evolution 56:2445-2458.

Associate Editor: Anurag Agrawal Editor: Mark A. McPeek 